\title{
Transitional care intervention effects on mortality and healthcare utilisation: a systematic review of pragmatic trials
}

\author{
Authors: Shawn CW Ng, ${ }^{\mathrm{A}}$ Yu H Kwan ${ }^{\mathrm{A}}$ and Lian L Low ${ }^{\mathrm{B}}$
}

\section{Introduction}

Transitions in care for patients with chronic conditions are often complex, leading to increased mortality and healthcare utilisation. Systematic reviews on effectiveness of transitional care (TC) interventions are inconclusive and none to date have examined pragmatic randomised controlled trials (RCTs) specifically. We aimed to determine the effectiveness of the components of TC interventions on mortality and healthcare utilisation.

\section{Materials and methods}

We conducted a systematic review to determine the effectiveness of TC interventions for patients suffering from one or more chronic diseases following the preferred reporting items for systematic reviews and meta-analyses criteria. RCTs were identified through PUBMED, Cochrane and EMBASE (1960-2018). Two independent reviewers performed the study selection, pragmatic scoring (using PRECIS-2 tool) and data extraction.

\section{Results and discussion}

Out of 13,918 articles, 37 pragmatic RCTs were reviewed. TC interventions improved mortality and/or hospital readmission in most of the articles ( $n=33$ ). Education, structured telephone support and home visits were the most commonly used and were a demonstrably effective intervention. Case management and discharge planning however showed poor evidence of effecting positive health outcomes. Medication reconciliation, in contrast to reviews that studied non-pragmatic trials, also showed poor evidence for positive health outcomes. To our best knowledge, this is the first review that examined the effectiveness of TC interventions in pragmatic trials, demonstrating the difference that an analysis of pragmatic trials, as opposed to explanatory trials, can have on health outcomes.

\section{Conclusion}

TC interventions improve transitions of care and should be incorporated in discharge processes, especially education, telephone and/or home visit follow-ups. Case management, discharge planning and medicine reconciliation however should

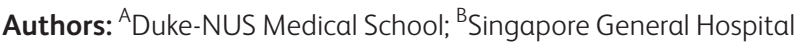

be applied with caution in designing future TC interventions. Additionally, future research into transitional care should take pragmatism into consideration to better inform policymakers and clinicians on its real world effects. 